\title{
Tumorigenesis via satellite link
}

Repetitive DNA sequences and additional non-protein-coding genomic regions have historically been excluded from transcriptomic analyses. However, the expression and functional importance of various non-coding regions is now increasingly being appreciated, and a new study has shown that the aberrant expression of satellite repeats has intriguing links to tumorigenesis.

Daniel Haber and colleagues used digital gene expression (DGE), an unbiased method of RNA deep sequencing, to characterize the transcriptomes of primary tumour samples. In a sample from a mouse pancreatic ductal adenocarcinoma (PDAC) that was driven by the expression of KRAS-G12D and the loss of $\operatorname{Tr} p 53$, nearly $50 \%$ of sequenced transcripts came from the pericentromeric (major) 234 base pair satellite repeats that are known to be required for heterochromatin formation and accurate chromosome segregation. Expression levels from this major satellite were more than 100 -fold greater than in the normal pancreas. In addition, although there was variability in its absolute expression level, the major satellite is selectively overexpressed (by a mean of 40 -fold) in other epithelial mouse cancers that are driven by different oncogenic lesions, compared with normal tissue counterparts. Genomic DNA sequencing confirmed that this upregulation is not caused by amplification.

Similar transcriptome analyses in human tumours from different tissues revealed that a subset of human satellite sequences, such as HSATII, were also upregulated in cancerous tissue in comparison to normal tissue.

Interestingly, despite being conserved across species, this mechanism seems to be restricted to in vivo settings. Immortalized cell lines that were established from satellite-overexpressing PDACs showed minimal satellite overexpression, although this repression could be reversed either by xenograft transplantation into nude mice or by treatment with the demethylating agent 5 -azacytidine. This suggests that the tumour microenvironment might influence the epigenetic regulation of satellite expression.

The authors also examined protein-coding sequences from the DGE studies to identify genes that are co-regulated with tumorigenic satellites. They identified genes that are involved in neural cell fate and germ cell and stem cell pathways. However, it remains unclear whether the expression of these genes will be more clinically informative than the expression of genes identified in other, satellite-independent tumour versus normal transcriptome studies.

The prognostic and diagnostic potential of satellite expression was shown by the observation of satellite expression in mouse pre-neoplastic pancreatic lesions, which increased during progression to advanced disease. Furthermore, in a pilot study of ten fine-needle aspirate clinical samples, satellite RNA in situ hybridization outperformed histopathological analysis for the diagnosis of human PDAC.
Although satellite expression has been associated with genomic instability, it is not yet clear whether this satellite deregulation is actively driving genomic instability and tumorigenesis, or whether it is merely a consequence of it. Nevertheless, satellite expression might become a valuable clinical biomarker for cancer detection and staging.

Darren J. Burgess

ORIGINAL RESEARCH PAPER Ting, D. T. et al. Aberrant overexpression of satellite repeats in pancreatic and other epithelial cancers. Science 331, 593-596 (2011)

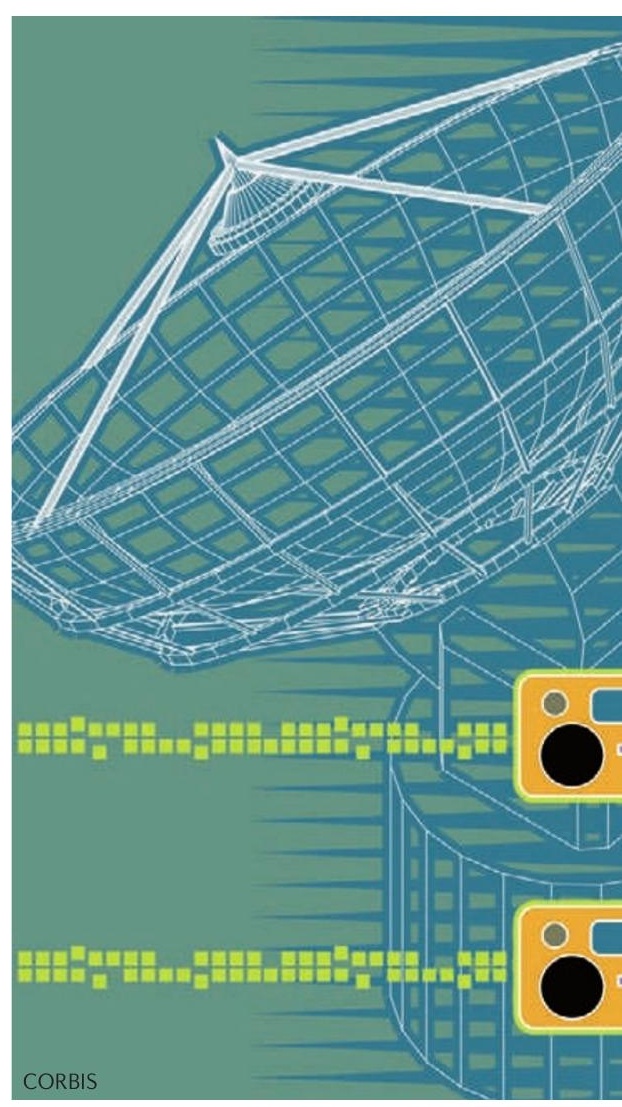

